\title{
Neural signature of error processing in major depression
}

\author{
Kathrin Malejko ${ }^{1}$ (1) Stefan Hafner ${ }^{1} \cdot$ Paul L. Plener $^{2,3} \cdot$ Martina Bonenberger $^{2} \cdot$ Georg Groen $^{1} \cdot$ Birgit Abler $^{1}$. \\ Heiko Graf ${ }^{1}$
}

Received: 15 October 2020 / Accepted: 3 February 2021 / Published online: 17 February 2021

(c) The Author(s) 2021

\begin{abstract}
The clinical presentation of major depression (MD) is heterogenous and comprises various affective and cognitive symptoms including an increased sensitivity to errors. Various electrophysiological but only few functional magnetic resonance imaging (fMRI) studies investigated neural error processing in MD with inconsistent findings. Thus, reliable evidence regarding neural signatures of error processing in patients with current MD is limited despite its potential relevance as viable neurobiological marker of psychopathology. We therefore investigated a sample of 16 young adult female patients with current MD and 17 healthy controls (HC). During fMRI, we used an established Erikson-flanker Go/NoGo-paradigm and focused on neural alterations during errors of commission. In the absence of significant differences in rates of errors of commission in MD compared to $\mathrm{HC}$, we observed significantly ( $p<0.05$, FWE-corrected on cluster level) enhanced neural activations of the dorsal anterior cingulate cortex (dACC) and the pre-supplementary motor area (pre-SMA) in MD relative to HC and thus, in brain regions consistently associated to neural error processing and corresponding behavioral adjustments. Considering comparable task performance, in particular similar commission error rates in MD and HC, our results support the evidence regarding an enhanced responsivity of neural error detection mechanisms in MD as a potential neural signature of increased negative feedback sensitivity as one of the core psychopathological features of this disorder.
\end{abstract}

Keywords Error processing $\cdot$ Commission errors $\cdot$ Major depression $\cdot$ Go/NoGo

\section{Introduction}

Major depression (MD) is one of the most commonly diagnosed mental disorders in first world countries affecting more than 264 million people worldwide [1]. The disorder is characterized by core symptoms of persistent feelings of depressed mood or loss of interest, and is diagnosed by behavioral observations according to the diagnostic criteria defined in the DSM-5 [2]. However, it is well known that the clinical presentation of MD is heterogenous and symptoms comprise a culmination of various affective and cognitive deficits. In particular, converging evidence suggests

Kathrin Malejko

kathrin.malejko@uni-ulm.de

1 Department of Psychiatry and Psychotherapy III, Ulm University Hospital, Leimgrubenweg 12-14, Ulm, Germany

2 Department of Child and Adolescent Psychiatry and Psychotherapy, Ulm University Hospital, Ulm, Germany

3 Department of Child and Adolescent Psychiatry, Medical University of Vienna, Vienna, Austria valence-specific maladaptions in MD, such as an excessive sensitivity to negative feedback and errors of commission [3-5], i.e. differences between intended and executed responses. This increased sensitivity may act as a crucial psychological factor in the development and maintenance of this disorder.

Electrophysiological studies have intensively investigated neurofunctional signatures of error processing. These studies inferred the existence of a generic, high-level human error processing system from the error-related negativity (ERN), a negative deflection in an event-related potential component elicited about 50-100 ms after erroneous responses [6-10]. The anterior cingulate cortex (ACC) and adjacent pre-supplementary motor area (pre-SMA) were identified as a core system in error processing and as neural generator of the ERN [11-14]. In addition, functional magnetic resonance imaging (fMRI) studies reliably demonstrated the involvement of the inferior frontal cortex, encompassing the frontal operculum and the anterior insula in error processing $[12,15-18]$. 
Various electrophysiological studies have also investigated neural error processing in MD, but revealed heterogenous findings regarding the amplitude of the ERN. In particular, studies using experimental tasks devoid of incentive manipulations, observed an elevated ERN in middle-aged and elderly patients with MD [19-21]. Other studies also demonstrated an increase in ERN amplitudes but only for errors after erroneous trials [22, 23]. However, no differences in ERN amplitudes in MD compared to healthy controls (HC) were observed within the context of reward [20], and other studies even found an attenuated ERN-amplitude in young patients with MD [24], or in severely depressed patients during commission errors following erroneous trials $[25,26]$.

Only few fMRI studies investigated the neurofunctional signature of error processing in MD so far. One fMRI study investigated neural error processing in patients with remitted MD compared to HC and demonstrated neural hypoactivity within the rostral ACC (rACC) and the dorsomedial prefrontal cortex (dmPFC) during commission errors [27]. A more recent fMRI-study in patients with remitted MD associated an attenuated neural activation within the middle frontal gyrus during commission errors with higher risk for depression recurrence [28]. Another fMRI-study mainly focused on neural activations of inhibitory control and corresponding treatment response, but investigated neural alterations of error processing in MD with a contextual parametric Go/NoGo-paradigm with three separate levels [29]. This study demonstrated an increase in neural activations within the medial frontal gyrus and the precuneus during errors of commission in MD relative to $\mathrm{HC}$.

Of note, the examination of neural responses to errors is thought to be a viable neurobiological marker of psychopathology [30-33] that holds promise towards improving diagnostic procedures and treatment outcome or monitoring. Furthermore, there is an ongoing search for neurobiological markers, that could have the potential to serve as predictors for pharmacological treatment response in tailoring individual pharmacotherapy to patients's needs. Considering the inconsistent findings regarding neural signatures of error processing in MD that presumably owe to different symptom state, symptom severity and experimental tasks, there is an inevitable need for replication and validation of neural responses of error processing in independent samples before these potential biomarkers can be translated into clinical practice. We therefore investigated a sample of patients with current major depression and non-depressive, healthy controls. During fMRI, we used an established modified Erikson-flanker Go/NoGo-paradigm without incentive manipulations, that has been shown to reliably elicit neural responses in brain areas corresponding to commission errors and error processing [34-37]. Based on previous electrophysiological and neuroimaging studies using non-rewarding tasks [19-21, 29], we expected an increased neural response in MD of brain regions previously related to error processing, and in particular of the ACC.

\section{Methods}

\section{Subjects}

As part of a broader research project with different experiments of which findings are reported elsewhere [38, 39], we investigated a total of 33 young adult female participants aged 18-38 years. Of those, 16 patients were diagnosed with MD whereas 17 healthy participants (HC) served as control group with no current or lifetime psychiatric diagnosis. Participants in the MD- and HC-group were matched for their highest degree of education. Of note, patients with $\mathrm{MD}$ were on average about 5 years older than HC (Table 1). Two subjects in the MD-group also met the diagnostic criteria of dysthymia according to DSM-5. One further subject in the MD-group had a history of anorexia that was remitted for several years at time of investigation. 14 patients had a recurrent depressive disorder with an average of around three $(\mathrm{SD}=2.08)$ episodes and two had a first depressive episode. Participants were recruited from inpatient and outpatient units of the Department of Psychiatry and Psychotherapy III of the Ulm University Hospital. All 33 participants were right-handed according to the Edinburgh Handedness Inventory [40]. Regular smoking cigarettes was reported from three of the MD- and four participants of the HC-group but was prohibited at least $2 \mathrm{~h}$ before fMRI-scanning. Participants with any severe medical disorder, epilepsy, psychotic disorder, substance use disorder or regular consumption of alcohol or illegal drugs were excluded from the study. Antidepressant medication was not interrupted except of sedative drugs on the day of investigation. Patients of the MD-group took antidepressant medication of various kinds (see Supplementary material Table S1). One patient had a concomitant medication with topiramate and another patient with pregabalin, which were paused for a wash-out period of at least 3 days prior to fMRI-scanning (corresponding to 5 times the half-life of the substances). All participants gave written informed consent prior to the study that was approved by the local ethical committee of Ulm University and conducted in accordance with the Declaration of Helsinki.

\section{Psychometric measurements}

All participants were screened by using the Structured Clinical Interview for DSM-IV [SCID-I and -II; [41]] and clinical diagnoses of patients with MD were verified by one of the study psychologists or physicians. Current depressive symptoms were assessed by a German version of the Beck 
Table 1 Demographical and behavioral data of healthy controls (HC) and patients with major depression (MD)

\begin{tabular}{lllll}
\hline & HC & MD & t-test & \\
\cline { 4 - 5 } & Mean (sem) & Mean (sem) & $t$ & $P$ \\
\hline Demographical data & & & & \\
Age (years) & $23.06(1.03)$ & $28.69(1.15)$ & -4.41 & 0.001 \\
Education (school years) & $10.82(0.41)$ & $10.81(0.42)$ & 0.02 & 0.985 \\
Smoking behavior & & & & \\
Subjects smoking cigarettes (n/total) & $4 / 17$ & $3 / 16$ & & \\
Cigarettes per day & $7(8.98)$ & $12(9.85)$ & & -1.84 \\
Psychometric measures & & & & \\
BDI & $3.24(0.96)$ & $33.63(2.87)$ & -11.86 & 0.000 \\
BIS & $57.76(1.91)$ & $65.25(2.04)$ & -3.25 & 0.012 \\
Incorrect incongruent NoGo trials & & & & \\
Errors of commission & $13.24(2.68)$ & $7.75(1.10)$ & 2.36 & 0.074 \\
Reaction time (ms) & $420.63(15.31)$ & $452.28(15.45)$ & -1.77 & 0.156 \\
\hline
\end{tabular}

Statistical analyses were conducted by two-sided unpaired samples t-tests. Significant results $(p<0.05)$ are highlighted in italic font

$B D I$ beck depression inventory, $B I S$ barratt impulsiveness scale, sem standard error of the mean, $m s$ milliseconds
Depression Inventory, [second edition, BDI-II; [42]]. To assess impulsivity as personality trait, we used the Barratt Impulsiveness Scale 11th revision [BIS-11; [43-45]], a selfreporting questionnaire composed of 30 items, each rated on a 4-point Likert scale [44]. Higher total sum-scores reflect higher trait impulsivity. Two-sided $t$-tests for unpaired samples were computed to analyze psychometric scales.

\section{fMRI paradigm}

During fMRI, we used a combined Erikson-flanker Go/ NoGo-paradigm [46], that has been established in several electrophysiological and fMRI studies on error processing [34-37]. Five-letter strings were presented comprising the letters R, U, P, and V, with the action-relevant target always mid-standing. During Go trials, subjects were asked to respond with their right index finger on a two-button box to the target letter $\mathrm{R}$ and with their right middle finger to the target letter U. In NoGo trials, subjects should withhold their response upon appearance of the letters $\mathrm{P}$ or $\mathrm{V}$. Target and flanker stimuli were combined either congruently or incongruently. In congruent trials, all five letters were the same. In incongruent Go trials, targets were flanked by visually similar NoGo target letters (e.g., VVUVV). In incongruent NoGo trials, the central NoGo target was flanked by visually similar Go targets (e.g., UUVUU). The trial ended with the presentation of feedback stimuli (see Fig. 1). To ensure that task conditions and, in particular, incongruent NoGo trials provided enough errors for the study of error-related signals, the instruction to the participants emphasized speed over accuracy. Combination of the factors condition (Go, NoGo) and type (congruent, incongruent) yielded 66 trials of each level, resulting in a total of 264 trials. One trial lasted $1.9 \mathrm{~s}$; mean intertrial interval was $3.01 \mathrm{~s}$. The average stimulusonset asynchrony for events of the same combination of condition by type (e.g., incongruent NoGo) was $19.5 \mathrm{~s}$. The duration of the whole task was 22 min. Reaction times and correctness of subjects` responses on each trial were registered by a standard personal computer.

\section{fMRI data acquisition}

Due to a scanner update during data acquisition, functional imaging data were obtained by a 3 T MAGNETOM Allegra (Siemens, Erlangen, Germany) for the HC-group, and by a 3 T MAGNETOM Prisma Scanner (Siemens, Erlangen, Germany) for the MD-group. A T2*-sensitive gradient echo sequence was used for functional imaging of both samples with an echotime (TE) of $33 \mathrm{~ms}$, a flip angle of $90^{\circ}$, a field of view (FOV) of $230 \mathrm{~mm}$, and a slice thickness of $2.5 \mathrm{~mm}$ with an interslice gap of $0.5 \mathrm{~mm}$. At a repetition time (TR) of $2000 \mathrm{~ms}, 35$ transversal slices were recorded with an image size of $64 \times 64$ pixels during the Go/NoGo task. High-resolution T1-weighted anatomical images were obtained using three-dimensional magnetization-prepared rapid acquisition with gradient echo sequences $(1 \times 1 \times 1 \mathrm{~mm}$ voxels, bandwidth (BW) $130 \mathrm{~Hz} /$ Pixel, TR $2500 \mathrm{~ms}$, TI $1.1 \mathrm{~s}$, echotime (TE) $4.57 \mathrm{~ms}$, flip angle $12^{\circ}$ ).

\section{fMRI-data analysis}

Image pre-processing and statistical analyses were carried out using Statistical Parametric Mapping (SPM12, Wellcome Department, London, UK) with a random effects model for 
Fig. 1 fMRI-task: Eriksonflanker Go/NoGo-paradigm. Presentation of each trial began with a centrally presented fixation cross for a period of $500 \mathrm{~ms}$. Afterward, letter strings were centrally shown for a duration of $200 \mathrm{~ms}$, followed by a blank screen with a duration of $700 \mathrm{~ms}$. The trial ended with the presentation of feedback stimuli. According to the subjects' performance, the German expressions for either correct or wrong were presented for $500 \mathrm{~ms}$. The figure depicts a sequence of an incongruent NoGo trial. The target letter ' $\mathrm{V}$ ' is highlighted by a red rectangle for demonstrational purposes

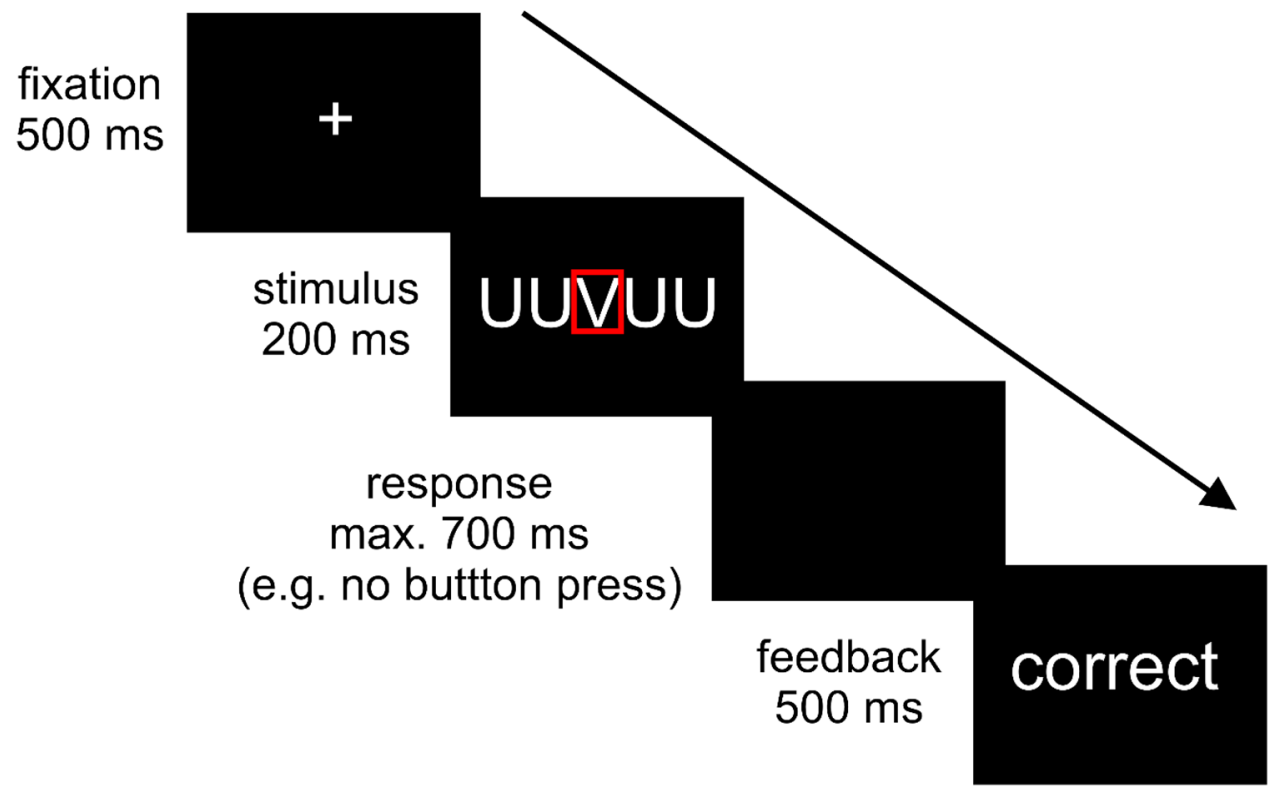

group analyses. Data from each session were pre-processed including slice-timing, realignment and normalization into a standard template (Montreal Neurological Institute, MNI) with a spatial resolution of $2 \times 2 \times 2 \mathrm{~mm}^{3}$. Smoothing was applied with an 8-mm FWHM isotropic Gaussian kernel. Intrinsic autocorrelations were accounted for by AR (1) and low frequency drifts were removed via high-pass filtering $(1 / 128 \mathrm{~s})$. On the single subject level, individual event types were modeled as trains of delta functions at each stimulus onset convolved with a canonical hemodynamic response function. Fixed-effects analyses comprised modeling of individual events following the combination of the factors condition (Go, NoGo), type (congruent, incongruent) and response (correct, incorrect), resulting in eight conditions. The six realignment parameters were added to the design matrix.

According to our previous studies conducted with the same task with the primary focus on error processing [34, 37], second level group analyses were computed within a $2 \times 2$ analysis of variances (ANOVA) with the two factors 'group' (HC, $\mathrm{MD}$ ) and 'condition' (NoGo/incongruent/correct, NoGo/ incongruent/incorrect). Congruent NoGo trials did not consistently yield errors across subjects and were therefore not further analyzed. Computation of between-groups differences in neural activations related to errors of commission, were constrained to voxels significant $(p<0.05)$ within a conjunction analysis consisting of differential neural activation (incorrect minus correct incongruent NoGo trials) of each of the two groups. Within this inclusive mask, group differences were computed for the contrast of incorrect minus correct incongruent NoGo trials. Here, significant group differences were inferred at a statistical threshold of $p<0.001$, uncorrected at the voxel level in combination with a minimum cluster size of 183 contiguously significant voxels, corresponding to an FWE-corrected $p$-value of $p<0.05$ at the cluster level. This specific number of 183 voxels was computed with a script from Tom Nichols and Marko Wilke (CorrClusTh.m v1.12 $2008 / 06 / 10$ ) that defines the extent of a cluster of contiguously significant (e.g. $p<0.001$ ) voxels, to survive a familywise corrected cluster-size $p$-value of $p<0.05$. The script can be found under https://warwick.ac.uk/fac/sci/statistics/staff/ academic-research/nichols/scripts/spm/johnsgems5/\#Gem6. Due to fMRI data acquisition on two different MRI-scanners and significant differences in age of the two groups (MD and $\mathrm{HC}$ ), scanner type and age were included as covariates in all fMRI-analyses. We also visually inspected one randomly selected EPI volume per each subject obtained from the Siemens MAGNETOM Allegra and the Siemens MANGETOM Prisma scanner, and found no indication for systematic differences in image quality. Moreover, only effects of condition differences but not of single conditions were allowed to enter computation group differences for statistical inference since only condition differences would compensate for putative, systematic contrast-to-noise scanner differences.

In case of significant group-by-condition interactions, individual differential neural activations (incorrect NoGo minus correct NoGo trials; averaged over significant voxels) in the MD-group only were correlated with individual numbers of errors, BDI and BIS scores by computing Pearson correlation coefficients. 


\section{Results}

\section{Demographical and behavioral data}

In line with the clinical diagnosis, significantly higher BDI sum-scores were observed in patients with MD relative to HC. In addition, we observed significantly higher BIS sumscores, measuring impulsivity as personality trait, in MD compared to HC. Regarding the behavioral responses in the Go/NoGo task during fMRI, reaction times and number of errors for incorrect incongruent NoGo trials did not significantly differ between MD and HC. Corresponding mean scores, $t$ - and $p$-values of the psychometric measurements and task responses in NoGo trials during fMRI are summarized in Table 1. In addition, we observed significant slower reaction times in correct Go trials in MD relative to $\mathrm{HC}$, whereas the number of correct responses in congruent and incongruent Go trials did not differ significantly between groups. Details on task responses in Go trials are provided in our supplementary material section (see Table S2).

\section{fMRI data}

A conjunction analysis comprising differential neural activations contrasting incorrect versus correct incongruent NoGo trials in each group, MD and HC, revealed significant $(p<0.05$; FWE-corrected on cluster level) differential neural activations within the right and left anterior insula, the right and left inferior frontal gyrus (IFG), the left supramarginal gyrus, the left dACC and the adjacent pre-SMA and thus, in brain regions that have been consistently associated to neural error processing. More details are provided in our supplemenatry material section (see Table S3 and Fig. S1).

Comparing both groups, we observed significant ( $p<0.05$; FWE-corrected on cluster level) higher differential (incorrect versus correct incongruent NoGo trials) neural activations due to commission errors in $\mathrm{MD}$ relative to HC within pre-SMA and dACC (see Table 2 and Fig. 2). No significantly higher differential neural activations were observed in HC compared to MD.

\section{Correlation analyses}

No significant correlations between individual numbers of errors, BDI and BIS scores and individual differential neural activations during commission errors were observed in the MD-group.

\section{Discussion}

Several electrophysiological and few fMRI studies have investigated neurofunctional alterations of error processing in MD. However, corresponding results are far from consistent. We investigated a sample of young female patients with MD and HC with fMRI and an established Erikson-flanker Go/NoGo-paradigm, to support reliable evidence for a neurofunctional signature of error processing in MD. Despite a comparable number of commission errors in MD relative to $\mathrm{HC}$, we observed a significant increase in error-related neural activations in MD within the $\mathrm{AACC}$ and the adjacent pre-SMA and thus in brain regions that were consistently associated to neural error processing.

According to the clinical diagnoses and similar to other studies [47-49], we observed significant higher BDI and BIS sum-scores in MD relative to HC. During the Eriksonflanker Go/NoGo-paradigm, the rate of commission errors in incongruent NoGo trials did not significantly differ between MD and HC. An equal or even a fewer rate of commission errors in MD is in line with previous observations [10, 24-26, 50] and has been interpreted as a cause of psychomotor retardation on the one hand, or a trade-off between accuracy and speed in MD on the other, in which responses are slower and perhaps more careful. Our finding of slower reaction times in correct and incorrect Go trials in MD and, although not statistically significant, slower reaction times and lower error rates in MD relative to $\mathrm{HC}$ in incongruent NoGo trials, is in line with this interpretation. Of note, a comparable rate of errors in both groups (MD and HC) may strengthen the interpretability of our fMRI results considering that neural alterations may not be confounded by an increased error rate [51].

Table 2 Significant ( $p<0.001, \mathrm{k}>183 \mathrm{Vx}$; FWE-corrected on cluster level) differential (incorrect minus correct incongruent NoGo trials) neural activations in patients with major depression (MD; $n=16)$ compared to healthy controls (HC; $n=17)$, with scanner type and age as covariates

\begin{tabular}{|c|c|c|c|c|c|c|c|c|}
\hline & \multirow[t]{2}{*}{$\mathrm{BA}$} & \multirow{2}{*}{$\begin{array}{l}\text { Anatomic } \\
\text { Label }\end{array}$} & \multirow[t]{2}{*}{$\mathrm{L} / \mathrm{R}$} & \multirow[t]{2}{*}{ Cluster size } & \multirow[t]{2}{*}{$\mathrm{Z}$} & \multicolumn{3}{|c|}{ MNI } \\
\hline & & & & & & $\mathrm{x}$ & $\mathrm{Y}$ & $\mathrm{Z}$ \\
\hline \multirow[t]{3}{*}{$\mathrm{MD}>\mathrm{HC}$} & 6 & Pre-SMA & $\mathrm{R}$ & 358 & 4.64 & 8 & 14 & 44 \\
\hline & & & $\mathrm{R}$ & & 4.21 & 8 & 6 & 56 \\
\hline & 24 & $\mathrm{dACC}$ & $\mathrm{L}$ & & 4.21 & -6 & 12 & 36 \\
\hline
\end{tabular}

$B A$ Brodman area, $L$ left, $R$ right, $M N I$ montreal neurological insitute (x-, y-, z-coordinates are provided in $\mathrm{mm})$; $Z$ z-score of standard norm distribution, $d A C C$ dorsal anterior cingulate cortex, pre-SMA pre-supplementary motor area 
Fig. 2 Significant differential (incorrect minus correct incongruent NoGo-trials) neural activations corresponding to errors of commission in patients with major depression (MD; $n=16)$ compared to healthy controls (HC; $n=17)$ masked within a conjunction analyses of differential (incorrect minus correct incruent NoGo trials) neural activations in $\mathrm{HC}$ and MD at a statistical threshold of $p<0.05$. Bar charts depict fMRI parameter estimates extracted from the significant cluster within the dorsal anterior cingulate cortex (dACC) and the pre-supplementary motor area (pre-SMA) for HC and MD; error bars is standard error of the mean. $H C$ healthy controls, $M D$ patients with major depression; iNoGo $+=$ correct incongruent NoGo-trials; iNoGo incorrect incongruent NoGotrials; iNoGo $\Delta$ incorrect minus correct incongruent NoGo-trials; $* * *=$ statistical significance $(p<0.05$, FWE-corrected on cluster level)
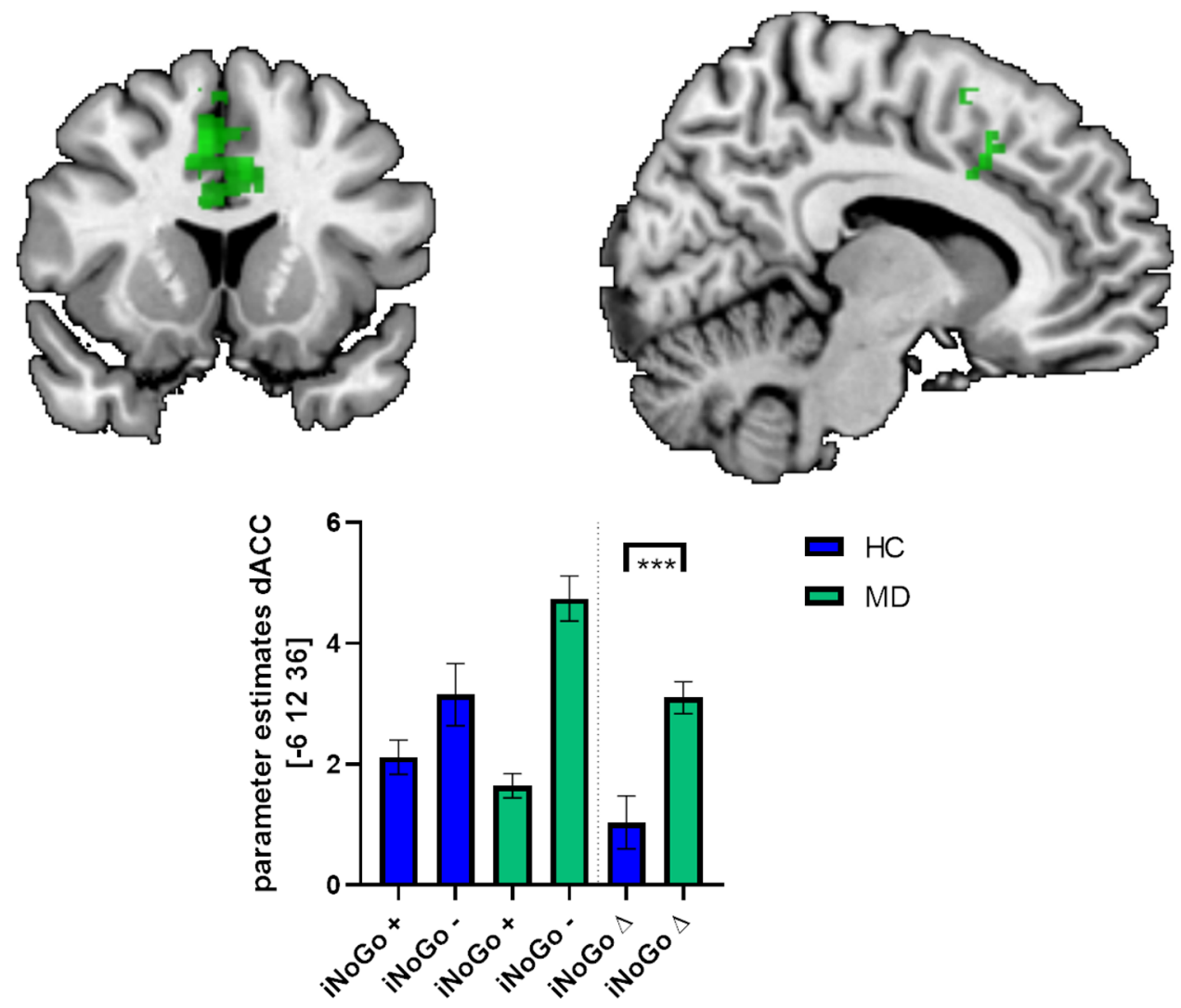

During fMRI, we observed differential neurofunctional activations related to commission errors within the dACC, pre-SMA, right and left IFG and anterior insula, as well as left supramarginal gyrus in HC and MD. Enhanced neural activations of these brain regions have been consistently associated with error processing [11, 12, 15-18, 52], supporting that our task induced reliable neural responses.

Comparing the two groups, MD and HC, we observed significantly enhanced differential neural activations related to errors of commission within the $\mathrm{AACC}$ and the pre-SMA in MD relative to $\mathrm{HC}$. The $\mathrm{dACC}$ responds to various types of performance feedback such as errors or conflicts [52]. Increased neural activations within this region were consistently associated with performance and error monitoring $[13,15,16,53,54]$. Beyond a pure monitoring function, the dACC also directs attention toward task-relevant stimuli and maintains associations between actions and their outcomes, including the implementation of task sets [52]. The neural signaling of the dACC may serve to improve task performance by modulating control over the motor system and allocations of capacities of different competing neural systems [9]. Our observation of enhanced neural activations within the dACC during commission errors in MD is also in line with several electrophysiological and one fMRI study [19-21, 29], that demonstrated an increased neural response pattern of this region during error processing in MD. By contrast, two other fMRI studies described diminished error-related neural activations of frontal brain areas including the ACC $[27,28]$. It is of note, however, that both studies investigated patients with remitted depressive disorder rather than patients with current MD as in our study. In addition, we observed significantly enhanced neural activations of the pre-SMA. Neural activations of this region have been associated with error detection $[6,8,13,59]$ and the identification of response conflicts [16, 54, 60, 61], with errors representing a special case of higher order conflict processing $[13,60]$. Beyond the observation that both, dACC and pre-SMA contribute to the ERN [11-14], there is evidence that these two regions response at different points in time. In particular, neural activations of the pre-SMA due to errors are thought to emerge earlier and to provide [62] and to precede [63] error-related signals as an input to the dACC. It has been also suggested that the pre-SMA reliably responds to negatively valenced signals that may arise from either error or conflict monitoring. In addition, neural signaling within the pre-SMA encodes the information to evaluate previous decision processes, necessary to learn from errors and to adjust erroneous behavior [64]. Unfortunately, we were not able to specifically investigate post-error adjustments as trial composition and stimulus arrays in our task are inappropriate for this purpose. 
With enhanced neural activations in error-related brain regions in the absence of statistically significant differences in error rates in MD compared to HC, our data support an increased responsivity of neural error detection in MD compared to HC. These enhanced neural responses may reflect a potential neural correlate of the clinically relevant and frequently observed enhanced sensitivity to negative environmental cues or errors [3-5]. However, a growing body of work indicates that also substance abuse is characterized by abnormal error-related neural responses of the ACC [55-58], potentially related to or mediated by increased impulsivity. This speaks against clinical specificity of our present observation. On the other hand, patients with substance disorder were not included in our study, and we also did not find a significant correlation between differential dACC/pre-SMA activations and individual BIS scores in MD.

Some shortcomings are to discuss. Our study was conducted in a relatively small sample size comprising only female participants. This may compromise the strength and the generalizability of our data, and present results await empirical replication with larger samples of both sexes. Moreover, our patients with MD were investigated under antidepressant medication that may have potentially altered neural activations relevant for error processing. However, similar error-related neural alterations in MD were also found in patients with MD without antidepressant medication [10]. Also, another electrophysiological study observed no differences in error-/event-related potential amplitudes between patients with MD with and without antidepressant medication [65]. As another limitation, mean age of patients with MD and controls differed significantly by about 5 years and patients were investigated with a different fMRI-scanner of the same manufacturer with the same field strength and acquisition parameter as HC. For compensation, age and scanner type were included as covariates in all fMRI-analyses. Also, visual inspection focusing on systematic differences in image quality between both MR scanners revealed no evidence that would speak against the comparability of fMRI activation patterns derived from both devices. We also found no hints for considerable differences in contrast-tonoise ratios between the two scanners, which is supported by the rather equivalence of neural signaling during incongruent correct NoGo trials in both groups as indicated in our Fig. 2. Finally, even if one MR scanner would have had a systematically greater contrast-to-noise ratio than the other, this signal gain would have affected both event types (correct and incorrect NoGo trials). Since the critical dependent variables were never signal changes against baseline but always signal changes between trials of different event types, any putative global signal differences are automatically taken into account by these condition differences used for between-group comparisons in our analysis.

\section{Conclusion}

The neural system for the identification of errors has been intensively investigated in healthy subjects by electrophysiology and functional neuroimaging studies. In major depression, the clinical observation of increased sensitivity to errors and negative feedback may suggest alterations in neural networks for error processing. However, reliable evidence for these neural alterations is limited by inconsistent findings despite their relevance as potential neurobiological marker of clinically relevant psychopathology. We therefore investigated a sample of female patients with current major depression by fMRI and an Erikson-flanker Go/NoGo-paradigm compared to HC. In the absence of significant differences in error rates of commission, we observed significantly enhanced neural activations of the AACC and the pre-SMA in MD relative to HC. These brain regions are well related to neural error processing. Present results therefore support the notion of enhanced responsivity of neural error processing mechanisms in MD as a potential neural signature of this disorder. Whether enhanced error-related recruitment of the dACC and pre-SMA is merely an expression of increased negative feedback responsibility of patients with depression or whether this phenomenon is functionally in service of ongoing behavioral adjustments, successful or unsuccessful, cannot be answered with present data and remains an open question for future studies in search of clinically relevant imaging biomarkers of depression.

Supplementary Information The online version contains supplementary material available at https://doi.org/10.1007/s00406-021-01238-y.

\section{Acknowledgements None.}

Author contributions $\mathrm{HG}$ and $\mathrm{KM}$ contributed substantially to the work, analyzed and interpreted the data, and drafted the manuscript. BA, SH, GG and PP contributed substantially to the work, interpreted the data, and revised the manuscript critically for important intellectual content. HG, KM and MB obtained the data. All the authors approved the final version to be published and agreed to be accountable for all aspects of the work in ensuring that questions related to the accuracy or integrity of any part of the work are appropriately investigated and resolved.

Funding Open Access funding enabled and organized by Projekt DEAL.

\section{Compliance with ethical standards}

Conflict of interest PP has received research funding from the German Federal Ministry of Education and Health (BMBF), the German Federal institute for Drugs and Medical Devices (BfARM), the Baden Wuerttemberg Foundation and the Volkswagen Foundation. He was involved in clinical research projects with Lundbeck and Servier. He received a speaker's honorarium from Shire. He declares no conflict 
of interest with regards to the work presented in this manuscript. The other authors have no conflicts of interest to declare.

Open Access This article is licensed under a Creative Commons Attribution 4.0 International License, which permits use, sharing, adaptation, distribution and reproduction in any medium or format, as long as you give appropriate credit to the original author(s) and the source, provide a link to the Creative Commons licence, and indicate if changes were made. The images or other third party material in this article are included in the article's Creative Commons licence, unless indicated otherwise in a credit line to the material. If material is not included in the article's Creative Commons licence and your intended use is not permitted by statutory regulation or exceeds the permitted use, you will need to obtain permission directly from the copyright holder. To view a copy of this licence, visit http://creativecommons.org/licenses/by/4.0/.

\section{References}

1. Disease GBD, Injury I, Prevalence C (2018) Global, regional, and national incidence, prevalence, and years lived with disability for 354 diseases and injuries for 195 countries and territories, 1990-2017: a systematic analysis for the Global Burden of Disease Study 2017. Lancet 392(10159):1789-1858. https:// doi.org/10.1016/s0140-6736(18)32279-7

2. American Psychiatric Association (2013) Diagnostic and Statistical Manual of Mental Disorders. 5th edn., Washington, DC

3. Elliott R, Sahakian BJ, Michael A, Paykel ES, Dolan RJ (1998) Abnormal neural response to feedback on planning and guessing tasks in patients with unipolar depression. Psychol Med 28(3):559-571. https://doi.org/10.1017/s0033291798006709

4. Steffens DC, Wagner HR, Levy RM, Horn KA, Krishnan KR (2001) Performance feedback deficit in geriatric depression. Biol Psychiatry 50(5):358-363. https://doi.org/10.1016/s00063223(01)01165-9

5. Fossati $P$ (2008) Neural signatures of cognitive and emotional biases in depression. Dialogues Clin Neurosci 10(3):358-361

6. Falkenstein M, Hohnsbein J, Hoormann J, Blanke L (1991) Effects of crossmodal divided attention on late ERP components. II. Error processing in choice reaction tasks. Electroencephalogr Clin Neurophysiol 78(6):447-455. https://doi.org/10. 1016/0013-4694(91)90062-9

7. Falkenstein M, Hoormann J, Christ S, Hohnsbein J (2000) ERP components on reaction errors and their functional significance: a tutorial. Biol Psychol 51(2-3):87-107. https://doi.org/10. 1016/s0301-0511(99)00031-9

8. Gehring WJ, Goss B, Coles MGH, Meyer DE, Donchin E (1993) A Neural system for error detection and compensation. Psychol Sci 4(6):385-390. https://doi.org/10.1111/j.1467-9280.1993. tb00586.x

9. Holroyd CB, Coles MGH (2002) The neural basis of human error processing: reinforcement learning, dopamine, and the error-related negativity. Psychol Rev 109(4):679-709. https:// doi.org/10.1037/0033-295X.109.4.679

10. Tang Y, Zhang X, Simmonite M, Li H, Zhang T, Guo Q, Li C, Fang Y, Xu Y, Wang J (2013) Hyperactivity within an extensive cortical distribution associated with excessive sensitivity in error processing in unmedicated depression: a combined event-related potential and sLORETA study. Int J Psychophysiol 90(2):282-289. https://doi.org/10.1016/j.ijpsycho.2013.09.001

11. Botvinick M, Nystrom LE, Fissell K, Carter CS, Cohen JD (1999) Conflict monitoring versus selection-for-action in anterior cingulate cortex. Nature 402(6758):179-181. https://doi. org/10.1038/46035
12. Debener S, Ullsperger M, Siegel M, Fiehler K, von Cramon DY, Engel AK (2005) Trial-by-trial coupling of concurrent electroencephalogram and functional magnetic resonance imaging identifies the dynamics of performance monitoring. J Neurosci 25(50):11730-11737. https://doi.org/10.1523/JNEUROSCI. 3286-05.2005

13. Taylor SF, Stern ER, Gehring WJ (2007) Neural systems for error monitoring: recent findings and theoretical perspectives. Neuroscientist 13(2):160-172. https://doi.org/10.1177/10738 58406298184

14. Dehaene S, Posner MI, Tucker DM (1994) Localization of a neural system for error detection and compensation. Psychol Sci 5(5):303-305

15. Braver TS, Barch DM, Gray JR, Molfese DL, Snyder A (2001) Anterior cingulate cortex and response conflict: effects of frequency, inhibition and errors. Cereb Cortex 11(9):825-836. https://doi.org/10.1093/cercor/11.9.825

16. Ullsperger M, von Cramon DY (2001) Subprocesses of performance monitoring: a dissociation of error processing and response competition revealed by event-related fMRI and ERPs. Neuroimage 14(6):1387-1401. https://doi.org/10.1006/nimg. 2001.0935

17. Garavan H, Ross TJ, Murphy K, Roche RA, Stein EA (2002) Dissociable executive functions in the dynamic control of behavior: inhibition, error detection, and correction. Neuroimage 17(4):1820-1829. https://doi.org/10.1006/nimg.2002.1326

18. Mathalon DH, Whitfield SL, Ford JM (2003) Anatomy of an error: ERP and fMRI. Biol Psychol 64(1-2):119-141. https://doi.org/10. 1016/s0301-0511(03)00105-4

19. Alexopoulos GS, Murphy CF, Gunning-Dixon FM, Kalayam B, Katz R, Kanellopoulos D, Etwaroo GR, Klimstra S, Foxe JJ (2007) Event-related potentials in an emotional go/no-go task and remission of geriatric depression. NeuroReport 18(3):217-221. https://doi.org/10.1097/WNR.0b013e328013ceda

20. Chiu PH, Deldin PJ (2007) Neural evidence for enhanced error detection in major depressive disorder. Am J Psychiatry 164(4):608-616. https://doi.org/10.1176/ajp.2007.164.4.608

21. Holmes AJ, Pizzagalli DA (2008) Spatiotemporal dynamics of error processing dysfunctions in major depressive disorder. Arch Gen Psychiatry 65(2):179-188. https://doi.org/10.1001/archg enpsychiatry.2007.19

22. Ruchsow M, Herrnberger B, Wiesend C, Gron G, Spitzer M, Kiefer M (2004) The effect of erroneous responses on response monitoring in patients with major depressive disorder: a study with event-related potentials. Psychophysiology 41(6):833-840. https://doi.org/10.1111/j.1469-8986.2004.00237.x

23. Ruchsow M, Herrnberger B, Beschoner P, Gron G, Spitzer M, Kiefer M (2006) Error processing in major depressive disorder: evidence from event-related potentials. J Psychiatr Res 40(1):3746. https://doi.org/10.1016/j.jpsychires.2005.02.002

24. Ladouceur CD, Slifka JS, Dahl RE, Birmaher B, Axelson DA, Ryan ND (2012) Altered error-related brain activity in youth with major depression. Dev Cogn Neurosci 2(3):351-362. https://doi. org/10.1016/j.den.2012.01.005

25. Schrijvers D, De Bruijn ER, Maas YJ, Vancoillie P, Hulstijn W, Sabbe BG (2009) Action monitoring and depressive symptom reduction in major depressive disorder. Int J Psychophysiol 71(3):218-224. https://doi.org/10.1016/j.ijpsycho.2008.09.005

26. Schrijvers D, de Bruijn ER, Maas Y, De Grave C, Sabbe BG, Hulstijn W (2008) Action monitoring in major depressive disorder with psychomotor retardation. Cortex 44(5):569-579. https://doi. org/10.1016/j.cortex.2007.08.014

27. Nixon NL, Liddle PF, Worwood G, Liotti M, Nixon E (2013) Prefrontal cortex function in remitted major depressive disorder. Psychol Med 43(6):1219-1230. https://doi.org/10.1017/S0033 291712002164 
28. Langenecker SA, Jenkins LM, Stange JP, Chang YS, DelDonno SR, Bessette KL, Passarotti AM, Bhaumik R, Ajilore O, Jacobs RH (2018) Cognitive control neuroimaging measures differentiate between those with and without future recurrence of depression. Neuroimage Clin 20:1001-1009. https://doi.org/10.1016/j.nicl. 2018.10.004

29. Langenecker SA, Kennedy SE, Guidotti LM, Briceno EM, Own LS, Hooven T, Young EA, Akil H, Noll DC, Zubieta JK (2007) Frontal and limbic activation during inhibitory control predicts treatment response in major depressive disorder. Biol Psychiatry 62(11):1272-1280. https://doi.org/10.1016/j.biopsych.2007.02. 019

30. Manoach DS, Agam Y (2013) Neural markers of errors as endophenotypes in neuropsychiatric disorders. Front Hum Neurosci 7:350. https://doi.org/10.3389/fnhum.2013.00350

31. Vaidyanathan U, Nelson LD, Patrick CJ (2012) Clarifying domains of internalizing psychopathology using neurophysiology. Psychol Med 42(3):447-459. https://doi.org/10.1017/S0033 291711001528

32. Weinberg A, Dieterich R, Riesel A (2015) Error-related brain activity in the age of RDoC: A review of the literature. Int J Psychophysiol 98(2 Pt 2):276-299. https://doi.org/10.1016/j.ijpsycho. 2015.02.029

33. Weinberg A, Liu H, Shankman SA (2016) Blunted neural response to errors as a trait marker of melancholic depression. Biol Psychol 113:100-107. https://doi.org/10.1016/j.biopsycho.2015.11.012

34. Graf H, Abler B, Freudenmann R, Beschoner P, Schaeffeler E, Spitzer M, Schwab M, Gron G (2011) Neural correlates of error monitoring modulated by atomoxetine in healthy volunteers. Biol Psychiatry 69(9):890-897. https://doi.org/10.1016/j.biopsych. 2010.10.018

35. Ruchsow M, Walter H, Buchheim A, Martius P, Spitzer M, Kachele H, Gron G, Kiefer M (2006) Electrophysiological correlates of error processing in borderline personality disorder. Biol Psychol 72(2):133-140. https://doi.org/10.1016/j.biopsycho.2005. 08.006

36. Ruchsow M, Spitzer M, Gron G, Grothe J, Kiefer M (2005) Error processing and impulsiveness in normals: evidence from eventrelated potentials. Brain Res Cogn Brain Res 24(2):317-325. https://doi.org/10.1016/j.cogbrainres.2005.02.003

37. Sosic-Vasic Z, Ulrich M, Ruchsow M, Vasic N, Gron G (2012) The modulating effect of personality traits on neural error monitoring: evidence from event-related FMRI. PLoS ONE 7(8):e42930. https://doi.org/10.1371/journal.pone.0042930

38. Malejko K, Neff D, Brown R, Plener PL, Bonenberger M, Abler B, Graf H (2018) Neural correlates of social inclusion in borderline personality disorder. Front Psychiatry 9:653. https://doi.org/10. 3389/fpsyt.2018.00653

39. Malejko K, Brown RC, Plener PL, Bonenberger M, Graf H, Abler B (2020) Differential neural processing of unpleasant sensory stimulation in patients with major depression. Eur Arch Psychiatry Clin Neurosci. https://doi.org/10.1007/s00406-020-01123-0

40. Oldfield RC (1971) The assessment and analysis of handedness: The Edinburgh inventory. Neuropsychologia 9(1):97-113. https:// doi.org/10.1016/0028-3932(71)90067-4

41. First MB, Spitzer RL, Gibbon M, Williams JB (1997) User's guide for the Structured clinical interview for DSM-IV axis I disorders SCID-I: clinician version. American Psychiatric Pub

42. Beck AT, Steer RA, Brown GK (1996) Manual for the beck depression inventory-II. San Antonio, TX: Psychological Corporation $1: 82$

43. Barratt E (1994) Impulsiveness and Aggression. Monahan J und Steadman H (Hrsg): Violence and Mental Disorder: Developments in Risk Assessment University of Chicago Press, Chicago S. $61-79$
44. Patton JH, Stanford MS, Barratt ES (1995) Factor structure of the Barratt impulsiveness scale. J Clin Psychol 51(6):768-774

45. Hautzinger M, Keller F, Kühner C (2006) Beck depressionsinventar (BDI-II). Harcourt Test Services Frankfurt

46. Eriksen BA, Eriksen CW (1974) Effects of noise letters upon the identification of a target letter in a nonsearch task. Percep Psychophys 16(1):143-149. https://doi.org/10.3758/bf03203267

47. Peluso MA, Hatch JP, Glahn DC, Monkul ES, Sanches M, Najt P, Bowden CL, Barratt ES, Soares JC (2007) Trait impulsivity in patients with mood disorders. J Affect Disord 100(1-3):227-231. https://doi.org/10.1016/j.jad.2006.09.037

48. Fortgang RG, Hultman CM, van Erp TG, Cannon TD (2016) Multidimensional assessment of impulsivity in schizophrenia, bipolar disorder, and major depressive disorder: testing for shared endophenotypes. Psychol Med 46(7):1497-1507. https://doi.org/10. 1017/S0033291716000131

49. Beier AM, Lauritzen L, Galfalvy HC, Cooper TB, Oquendo MA, Grunebaum MF, Mann JJ, Sublette ME (2014) Low plasma eicosapentaenoic acid levels are associated with elevated trait aggression and impulsivity in major depressive disorder with a history of comorbid substance use disorder. J Psychiatr Res 57:133-140. https://doi.org/10.1016/j.jpsychires.2014.06.012

50. Olvet DM, Klein DN, Hajcak G (2010) Depression symptom severity and error-related brain activity. Psychiatry Res 179(1):30-37. https://doi.org/10.1016/j.psychres.2010.06.008

51. Schroder HS, Moser JS (2014) Improving the study of error monitoring with consideration of behavioral performance measures. Front Hum Neurosci 8:178. https://doi.org/10.3389/fnhum. 2014.00178

52. Dosenbach NU, Visscher KM, Palmer ED, Miezin FM, Wenger KK, Kang HC, Burgund ED, Grimes AL, Schlaggar BL, Petersen SE (2006) A core system for the implementation of task sets. Neuron 50(5):799-812. https://doi.org/10.1016/j. neuron.2006.04.031

53. Carter CS, Macdonald AM, Botvinick M, Ross LL, Stenger VA, Noll D, Cohen JD (2000) Parsing executive processes: strategic vs. evaluative functions of the anterior cingulate cortex. Proc Natl Acad Sci U S A 97(4):1944-1948. https://doi.org/10.1073/pnas. 97.4.1944

54. Carter CS, Braver TS, Barch DM, Botvinick MM, Noll D, Cohen JD (1998) Anterior cingulate cortex, error detection, and the online monitoring of performance. Science 280(5364):747-749. https://doi.org/10.1126/science.280.5364.747

55. Castelluccio BC, Meda SA, Muska CE, Stevens MC, Pearlson GD (2014) Error processing in current and former cocaine users. Brain Imag Behav 8(1):87-96. https://doi.org/10.1007/ s11682-013-9247-y

56. Kaufman JN, Ross TJ, Stein EA, Garavan H (2003) Cingulate hypoactivity in cocaine users during a GO-NOGO task as revealed by event-related functional magnetic resonance imaging. J Neurosci 23(21):7839-7843

57. Steele VR, Fink BC, Maurer JM, Arbabshirani MR, Wilber CH, Jaffe AJ, Sidz A, Pearlson GD, Calhoun VD, Clark VP, Kiehl KA (2014) Brain potentials measured during a Go/NoGo task predict completion of substance abuse treatment. Biol Psychiatry 76(1):75-83. https://doi.org/10.1016/j.biopsych.2013.09.030

58. Claus ED, Shane MS (2018) dACC response to presentation of negative feedback predicts stimulant dependence diagnosis and stimulant use severity. Neuroimage Clin 20:16-23. https://doi.org/ 10.1016/j.nicl.2018.05.007

59. Falkenstein M, Hohnsbein J, Hoormann J (1995) Event-related potential correlates of errors in reaction tasks. Electroencephalogr Clin Neurophysiol Suppl 44:287-296

60. Botvinick MM, Braver TS, Barch DM, Carter CS, Cohen JD (2001) Conflict monitoring and cognitive control. Psychol Rev 108(3):624-652. https://doi.org/10.1037/0033-295x.108.3.624 
61. Kerns JG, Cohen JD, MacDonald AW 3rd, Cho RY, Stenger VA, Carter CS (2004) Anterior cingulate conflict monitoring and adjustments in control. Science 303(5660):1023-1026. https:// doi.org/10.1126/science. 1089910

62. Fu Z, Wu DJ, Ross I, Chung JM, Mamelak AN, Adolphs R, Rutishauser U (2019) Single-neuron correlates of error monitoring and post-error adjustments in human medial frontal cortex. Neuron. https://doi.org/10.1016/j.neuron.2018.11.016

63. Bonini F, Burle B, Liegeois-Chauvel C, Regis J, Chauvel P, Vidal F (2014) Action monitoring and medial frontal cortex: leading role of supplementary motor area. Science 343(6173):888-891. https://doi.org/10.1126/science.1247412

64. Behrens TE, Woolrich MW, Walton ME, Rushworth MF (2007) Learning the value of information in an uncertain world. Nat Neurosci 10(9):1214-1221. https://doi.org/10.1038/nn1954

65. Schoenberg PL (2014) The error processing system in major depressive disorder: cortical phenotypal marker hypothesis. Biol Psychol 99:100-114. https://doi.org/10.1016/j.biopsycho.2014.03. 005 